\title{
LA TALLA LITICA EN ATAPUERCA (BURGOS)
}

\author{
POR \\ MARINA MOSQUERA MARTINEZ (") \\ EUDALD CARBONELL I ROURA (“)
}

RESUMEN Este trabajo presenta fundamentalmente un estudio de los núcleos (BN1G neutras) recuperados en las excavaciones de los yacimientos mesopleistocenos de Atapuerca (Burgos). Se analizan y estudian los métodos de talla utilizados y las relaciones técnicas que existen entre éstos y sus productos, las lascas (BP). Por otra parte, se pretende una aproximación a las diferencias diacrónicas observadas en los rellenos, que, en alguno de los yacimientos, cubren la práctica totalidad del Pleistoceno Medio. Por último, se extrae una serie de conclusiones de tipo tecnológico que nos ayudan a caracterizar el período registrado en los yacimientos.

ABSTRACT This paper presents a study of the cores (neutral BN1G) from the Atapuerca Middle Pleistocene sites (Burgos, Spain). An analysis and study of the Atapuerca knapping methods is made, as well as one of the relationship between the knapping methods and the by-products (flakes, BP). On the other hand, an approach to the diachronic differences observed in the stratigraphies is outlined. Finally, some general conclusions are reached about the technology, in order to characterize the period recorded at these sites.

Palabras clave Núcleos. Métodos de talla. Atapuerca. Pleistoceno Medio.

Key words Cores. Knapping methods. Atapuerca. Middle Pleistocene.

\section{INTRODUCCION}

La Sierra de Atapuerca se encuentra situada a $15 \mathrm{~km}$. al Este de la ciudad de Burgos (Fig. 1). El río Arlazón, perteneciente a la cuenca del Duero, atraviesa la región de Este a Oeste hasta Burgos capital y discurre a unos $3 \mathrm{~km}$ al Sur del borde meridional de la Sierra. Sus coordenadas son: $40^{\circ}$ $21^{\prime} \mathrm{N}$ y $0^{\circ} 10^{\prime} \mathrm{E}$ del meridiano de Madrid y se sitúa en los términos municipales de Ibeas de Juarros, Atapuerca y Zalduendo.

(") Becaria F.P.I. Comunidad de Madrid. Dpto. de Paleontología. Museo Nacional de Ciencias Naturales. Madrid.

(“) Profesor titular. Laboratorio de Arqueología de la Universidad de Barcelona en Tarragona (L.A.U.B.T.). Facultad de Filosofia y Letras. Tarragona. 
Topográficamente es una suave elevación alargada y arqueada (Lám. I. A), constituida principalmente por calizas del Cretácico Superior que buzan al SW y W en la mitad meridional - en la que se desarrolla el karst donde se sitúan los yacimientos-, y por un anticlinal de dirección E-W donde afloran areniscas y gravas del Cretácico Inferior, en el extremo septentrional. El terreno circundante a la Sierra se compone fundamentalmente de arcillas, margas y rañas neógenas, características de toda la cuenca y de las llanuras aluviales cuaternarias de los cursos fluviales (Zazo et alii, 1987).

Los yacimientos de la Sierra de Atapuerca (Aguirre et alii, 1987) se encuentran en cavidades kársticas. Podemos distinguir dos grupos: yacimientos de la Trinchera y los de Cueva Mayor (Fig. 2). Los primeros son cavidades actualmente al descubierto, debido a la construcción a principios de este siglo de una línea de ferrocarril, poco después desmantelada. La Cueva Mayor parece haber sido objeto de ocupaciones pre y protohistóricas entre las que destaca la mesopleistocena, avalada por el hallazgo en la llamada Sima de los Huesos de, al menos, 17 individuos (Bermúdez de Castro, comunicación personal) (1) de la línea filogenética anteneandertal (Rosas, 1987; Rosas et alii, 1991). De ellos, y siguiendo al primer autor, uno es infantil, cuatro son juveniles o adolescentes, siete son

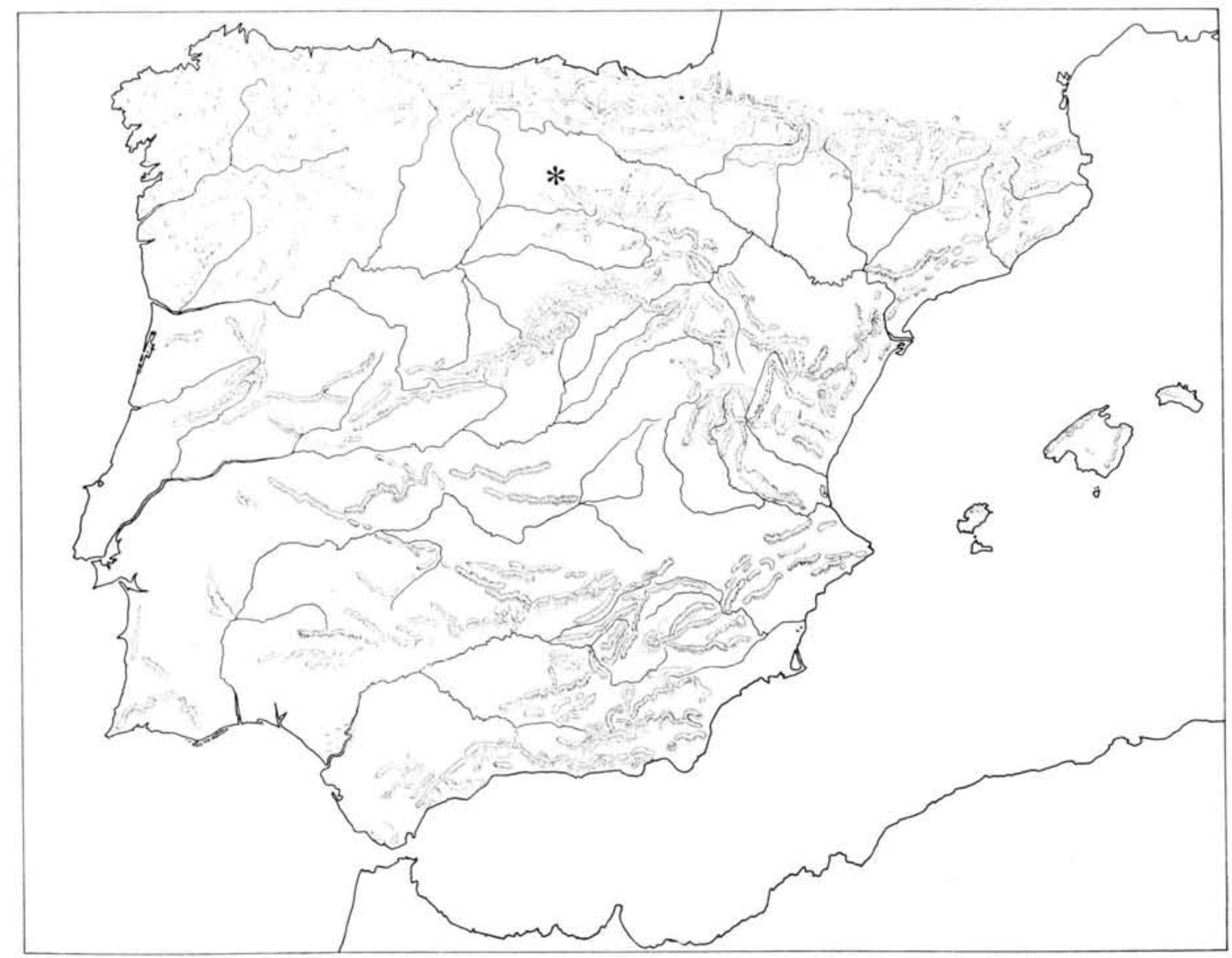

Fig. 1.-Situación aproximada de la Sierra de Atapuerca en el mapa general de la Península Ibérica.

(1) J. M. Bermúdez de Castro es colaborador cientifico en el Museo Nacional de Ciencias Naturales e Investigador Principal del provecto de investigación: "Evolución humana y ecosistemas en el Pleistoceno de la Sierra de Atapuerca» (DGCYT, PB900126-603). Es especialista en dentición de hominidos fósiles.

\section{T. P., 1992, nำ 49}



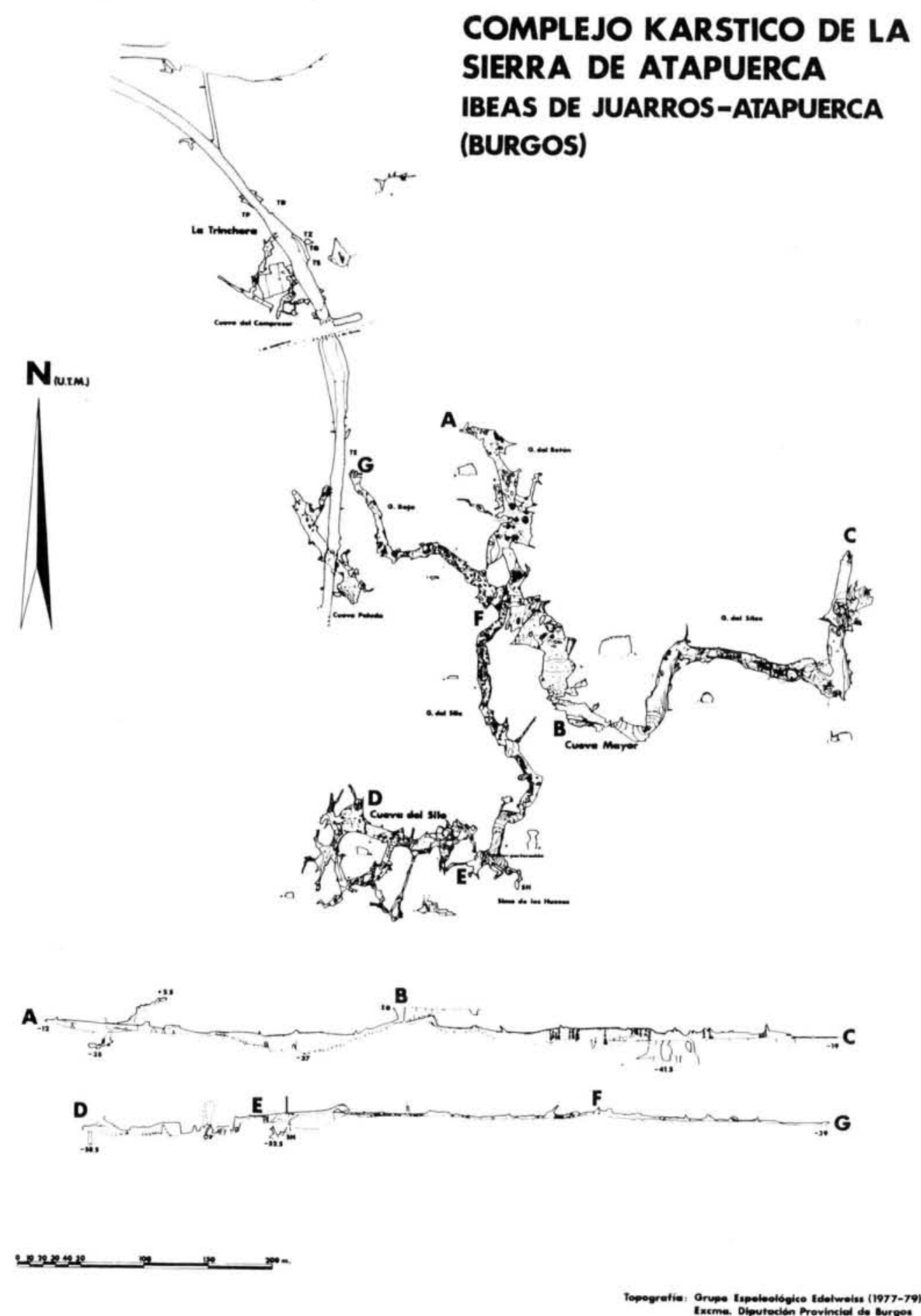

FIG. 2.-Complejo cárstico de la Sierra de Atapuerca. Al norte, la Trinchera con los yacimientos de Penal (TP), Gran Dolina (TD), Galería (TG), Covacha de los Zarpazos (TZ), Tres Simas (TS) y Elefante (TE). A sur, en la Cueva del Silo, el yacimiento de la Sima de los Huesos (SH), donde han sido localizados los fósiles humanos (Topografía: Grupo Espeleológico Edelweisss (1977-79). Excma. Diputación Prov. de Burgos). 
adultos jóvenes y cinco son adultos. Se ha podido determinar el sexo para catorce de esos individuos, de tal manera que habria nueve hembras y cinco machos. El origen de esta extraordinaria acumulación esta aún por dilucidar. Asociados a los restos humanos se hallan seis especies de carnivoros, contrastando con la ausencia total de herbivoros y de restos de actividades humanas.

La primera excavación fue realizada por el Prof. F. Jordá, T. Torres y N. Sánchez a finales de la década de los años 60, sobre lo que hoy denominamos TG. En 1978 el Prof. E. Aguirre retoma la excavación del yacimiento, ampliando las prospecciones, muestreos y excavaciones a todo el área de la Trinchera del Ferrocarril, y organiza un equipo de investigación constituido por arqueólogos, paleontólogos y geólogos, que continúa los trabajos hasta la actualidad. A partir de los primeros años quedaron localizados los siguientes yacimientos:

- Gran Dolina (TD) y el Penal (TP) que corresponden a un mismo conducto, actualmente dividido por el vaciado de la obra férrea.

- Complejo Tres Simas (TS), formado por Galería (TG), Tres Simas Boca Norte (TN) y Covacha de los Zarpazos (TZ), todas pertenecientes a la misma cavidad.

- Sima del Elefante (TE).

Hoy por hoy, los rellenos mejor conocidos son los de TD y TG-TN. El primero alcanza una potencia de $19 \mathrm{~m}$. El depósito de la Dolina (Lám. I. B) está dividido en 4 unidades sedimentarias y 11 niveles - numerados de base a techo-, la mayor parte fosiliferos y con restos arqueológicos a partir de TD4, muy cercano al límite Matuyama/Brunhes. A unos $4 \mathrm{~m}$. de la base conocida (TD3) se halla el primer depósito fosilifero, correspondiente a complejos Cromerienses. En él se ha detectado la inversión paleomagnética Matuyama/Brunhes, que marca la transición convencional entre el Pleistoceno inferior y el medio ( \pm 700.000 años). El último depósito (TD11) parece responder a las fases finales del Pleistoceno medio.

El otro gran depósito lo constituye el complejo TZ-TG-TN (Lám. II. A) situado a unos $25 \mathrm{~m}$. de TD, en el cual los depósitos horizontales de TG provienen, según las fases, de TZ - en la actualidad sólo explorada- y del conducto vertical TN. La potencia de esta última es de 13,5 m. y la de TG es de 4 a $6.5 \mathrm{~m}$. La cavidad TG-TN permanece cerrada durante gran parte del Pleistoceno Medio, abriéndose tras la formación de un complejo estalagmítico (TG4) datado en $-317.6 \pm 60 \mathrm{ka}$ (ESR) y $>=350$ ka de U-Series (Grün y Aguirre, 1987). Al parecer, los depósitos terminales de TG (TG12) se datan según los mismos autores en $177.3 \pm 23 \mathrm{ka}$ (ESR) y $118+71 /-79 \mathrm{ka}$ (familia de Uranio). A partir de TN3 encontramos depósitos fosiliferos y, con algún vacío, arqueológicos. En la Tabla I se muestra un cuadro provisional de las correlaciones entre los diferentes niveles de cada depósito (Aguirre, com. pers.).

Tras la primera excavación de Jordá, Torres y Sánchez, la extensión a excavar en TG quedó reducida a unos $18 \mathrm{~m}^{2}$. Son estériles algo más de $1 \mathrm{~m}$. superior y los $6 \mathrm{~m}$. superiores de TN (Bermúdez de Castro, 1990). A la espera de estudios más profundos y especializados, actualmente en realización, se ha de considerar la existencia de 13 suelos de utilización humana en TG-TN (Lám. II. B), de $25 \mathrm{~m}^{2}$ de extensión prácticamente horizontal, y 1 suelo de utilización en $\mathrm{TZ} \mathrm{de} 14 \mathrm{~m}^{2}$ de extensión (Carbonell et alii, 1987a) (2). En el caso de TG-TN, tanto el sedimento como la posición de los restos refleja un escaso o poco significativo movimiento post-deposicional. Se trata de asociaciones faunísticas e industriales en las que el escaso número de efectivos líticos, la gran cantidad de restos óseos - principalmente de ciervo, dominando los jóvenes, y caballos, representado en todas las edades- unido a la escasez de huellas de descarnado, todo ello hace pensar en un aprovechamiento faunístico puntual y en una presencia humana de corta duración y reiterada (Díez Fernández-

(2) La publicación citada recoge ochos suelos de utilización: los otros cinco han sido excavados posteriormente y están en vias de estudio.

T. P., 1992, $\mathrm{n}^{\circ} 49$ 
Lomana, com. pers.) (3). El último de los yacimientos de la Trinchera es la Sima del Elefante (TE), que es un conducto vertical situado a unos $300 \mathrm{~m}$. al sur de los yacimientos anteriormente mencionados. En él se han realizado muestreos para su datación geocronológica y recogida de fósiles y piezas líticas expuestas por la erosión en el corte (Bermúdez de Castro, 1990).

TABLA I

Esquema provisional de las correlaciones entre los diferentes niveles de cada depósito en los yacimientos de la Trinchera (Aguirre, com. pers.).

\begin{tabular}{|c|c|c|c|c|c|}
\hline & & & costra estalagm & & 117.000 \\
\hline & TD.11 & TG.12 & & TN.9 & \\
\hline & & TG.11 & & TN.8 & \\
\hline & & TG.10a & & TN.7 & \\
\hline & & & & costra & 250.000 \\
\hline & TD.10 & TG.10 & & TN.6 & \\
\hline & TD.9 & ? TG.9 & & & \\
\hline & & TG.8 & espeleot. & TN.5 & 280.000 \\
\hline & & TG.7 & & TN.4 & \\
\hline & TD. 8 & ? TG.6 & & TN.3 & \\
\hline & & TG.5 & & TN.2 & \\
\hline & & & & TN.1 & \\
\hline & & costra & estalagmitica & & $\geq 300.000$ \\
\hline & TD. 8 & ? TG.4 & & & \\
\hline & & TG.3 & & & \\
\hline \pm 400.000 & TD.7 & TG.2 & & & \\
\hline & TD.6 & TG.1 & & & \\
\hline & TD.5 & $?$ & & & \\
\hline & TD. 4 & & & & \\
\hline $690.000 / 730.000$ & TD. 3 & & & & \\
\hline & TD. 2 & & & & \\
\hline & TD.1 & & & & \\
\hline
\end{tabular}

\section{MATERIA PRIMA, CATEGORIAS ESTRUCTURALES Y YACIMIENTOS}

Para la comprensión tanto del conjunto industrial como de los suelos de utilización humana de los yacimientos, conviene recordar que parte de los depósitos fueron vaciados y puestos al descubierto por la antigua construcción del ferrocarril. Esto significa que grandes áreas de los depósitos . originales han sido destruidas, por lo que hoy por hoy no podemos evaluar su importancia o marginalidad respecto al total de los que se conservan. Un hecho a destacar es la relativa escasez de industria recuperada en las excavaciones, probablemente relacionado con la funcionalidad de las ocupaciones (Carbonell et alii, 1987a). Contamos con un conjunto de 733 piezas, de las cuales 353 pertenecen a TG-TN, 348 a TD y el resto (32 se distribuye entre los muestreos realizados en los otros yacimientos (TZ, TP, TE y Trinchera), sin determinar.

En este trabajo nos referiremos exclusivamente al material recuperado en tres de los yacimientos: Complejo Galería-Tres Simas (TG-TN) y Gran Dolina (TD). El material (701 piezas) se ha diferenciado por grandes paquetes de niveles sedimentarios:

(3) C. Diez Fdez.-Lomana forma parte del equipo investigador de Atapuerca desde 1980 y es especialista en estudios zooarqueológicos, tema principal de la Tesis Doctoral que recientemente ha presentado. 
1. Niveles superiores de TD (TD10 y 11) y sus correlativos en TG (TG10-12) y en TN (TN6-9); este conjunto ha sido excavado sistemáticamente desde 1981 y de él proviene la mayor parte de la industria (656 piezas). Se corresponde con un momento final del Pleistoceno Medio.

2. Niveles medios-superiores de TD (TD7-9) con sus correlativos en TG (niveles inferiores sin determinar-nivel TG9) y en TN (TN1-5). Se trata de 9 piezas procedentes de los niveles TG5/6 y TN2. Se calcula una edad entre 400.000 y 250.000 años.

3. Niveles medios-inferiores de TD (TD5 y 6). La caida de un bloque de sedimento correspondiente a estos niveles proporcionó un conjunto de 26 piezas y puso al descubierto otras 6 en el corte. La cronologia general de este conjunto se calcula en torno a los 450.000 años.

4. Niveles inferiores de TD (TD1-4), posiblemente sin paralelo en TG-TN. La excavación del nivel TD4 -inmediatamente por encima del límite Matuyama/Brunhes (Carracedo et alii, 1987) - a partir de la campaña de 1990 ha proporcionado otras 4 piezas líticas. Se estima una cronología aproximada a 650.000 años.

El estudio de la industria lítica de Atapuerca se realiza mediante el Sistema Analítico (Carbonell et alii, 1983; Carbonell, 1987, Carbonell et alii, 1987b y Carbonell et alii, 1992), que ofrece varios niveles de estudio, entre los que se encuentra el llamado nivel estructural, que sitúa a la pieza en su cadena de producción, asignándole una categoría estructural, dependiendo de la posición en la que se encuentre. La Fig. 3 nos ilustra sobre la cadena de producción y la formación de las cinco categorias estructurales fundamentales.

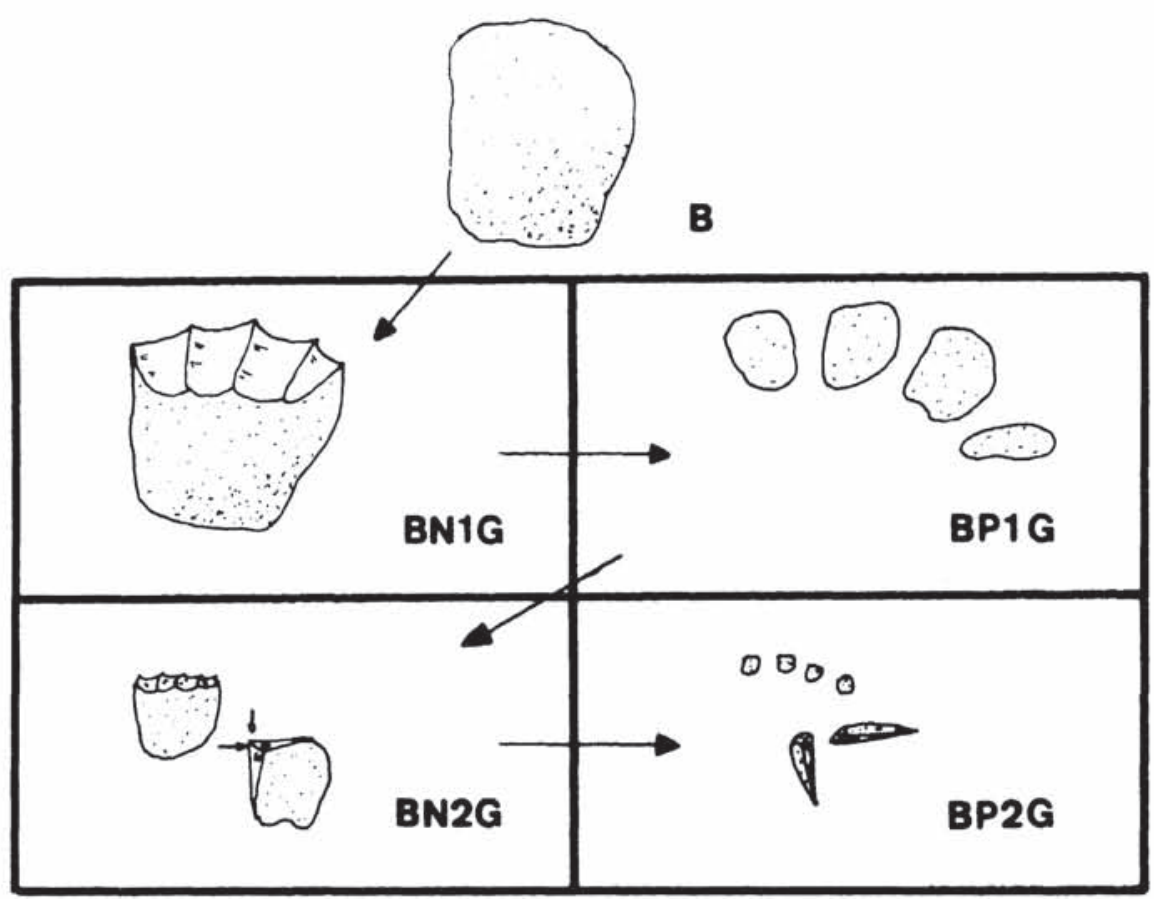

Fig. 3.-Primer nivel de análisis en el Sistema Analitico. Formación de categorias estructurales a partir de la situación que ocupa cada objeto en la cadena de producción. 
Estas categorías pueden definirse de la siguiente manera:

B: Bases sin modificar; manuports.

BN1G: Bases Negativas de Primera Generación; son Bases modificadas. Pueden ser núcleos (BN1G neutras) o grandes útiles, como triedros, etc. Los choppers y chopping-tools pertenecen también a esta categoría.

BP1G: Bases Positivas de Primera Generación: positivos o lascas obtenidas durante la talla de una BN1G.

BN2G: Bases Negativas de Segunda Generación; son Bases Positivas retocadas: raederas, raspadores, buriles, etc.

BP2G: Bases Positivas de Segunda Generación: positivos, lascas o esquirlas que se obtienen durante la talla o retoque de una BN2G.

En este trabajo no trataremos las BP2G, por lo que nos referiremos a las BP1G como BP, simplemente. Por otra parte, nos hemos visto obligados a incluir el grupo de Fragmentos Indeterminados, debido al mal estado de conservación en el que se encuentra gran parte de la industria de Atapuerca.

La distribución y representación de las categorías estructurales por yacimientos quedan reflejadas en la Tabla II. Existe un claro predominio en todos los yacimientos estudiados de las Bases Positivas (BP), seguidas de la BN2G o lascas modificadas (Fig. 4A) (4).

\section{TABLA II}

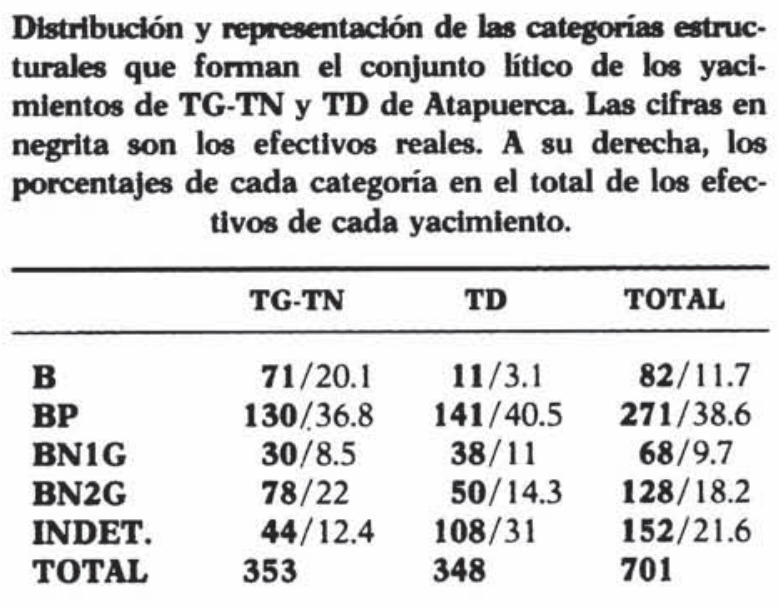

La utilización de sílex como materia prima es masiva $(61,7 \%)$ en relación a la cuarcita $(23,6 \%)$, arenisca (10\%), cuarzo $(2,1 \%)$ y caliza $(1,8 \%)$ (Fig. 4B).

El sílex presenta fundamentalmente dos modalidades: sílex errático y nódulos de cueva. El primero se presenta en grandes bloques diseminados por las antiguas terrazas del río Arlanzón (5). Este tipo de sílex se presenta generalmente en mal estado de conservación, incluso irrecuperable en algunas ocasiones. El $94 \%$ de los objetos de sílex corresponden a este tipo. La otra modalidad, utilizada en una baja proporción (6\%), es silex nodular, de una textura muy fina y de buena calidad, que se encuentra con relativa abundancia en afloramientos de algunas cuevas cercanas (Galería del Sílex en Cueva Mayor).

El predominio de sílex errático se hace patente en todas las categorías estructurales (Tabla III),

(4) Se excluye el grupo de Indeterminados.

(5) Para una visión geomorfológica de la zona, ver Zazo et alii, 1987. 
excepto en las Bases (B), que lógicamente corresponden a materiales más resistentes, como la cuarcita. De hecho, se ha documentado la existencia en muchas de estas Bases de fracturas y signos erosivos provocados probablemente por su utilización como percutores y machacadores.

Pese al reducido número de efectivos, se han podido observar una serie de pautas claras en la confección de los objetos líticos de Atapuerca. El análisis de estas pautas se ha realizado sobre las Bases Positivas, Bases Negativas de Primera Generación y Bases Negativas de Segunda Generación. Las BP y BNIG son las categorias estructurales que mantienen una relación más estrecha y directa entre si y con el tema tratado, pues entre las dos contienen toda la información sobre la talla. Las BN2G, por su parte, pueden conservar la información referente a las características del soporte (BP) sobre el cual están hechas. Sólo en este sentido son analizadas aqui.

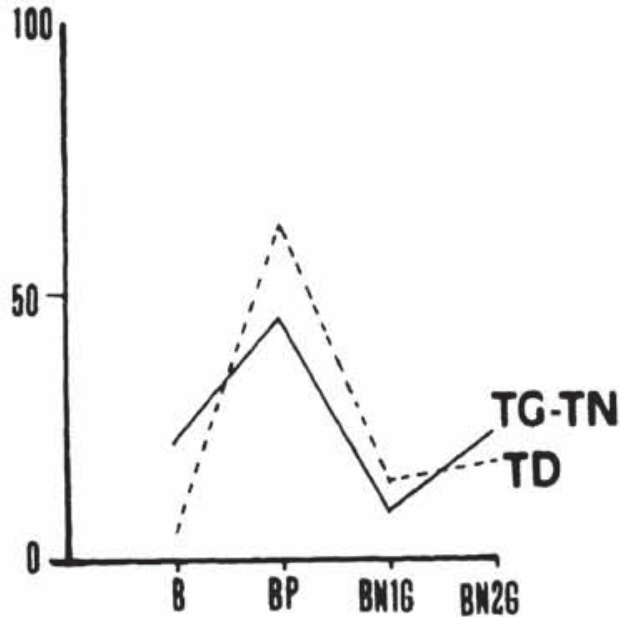

Figura 4A

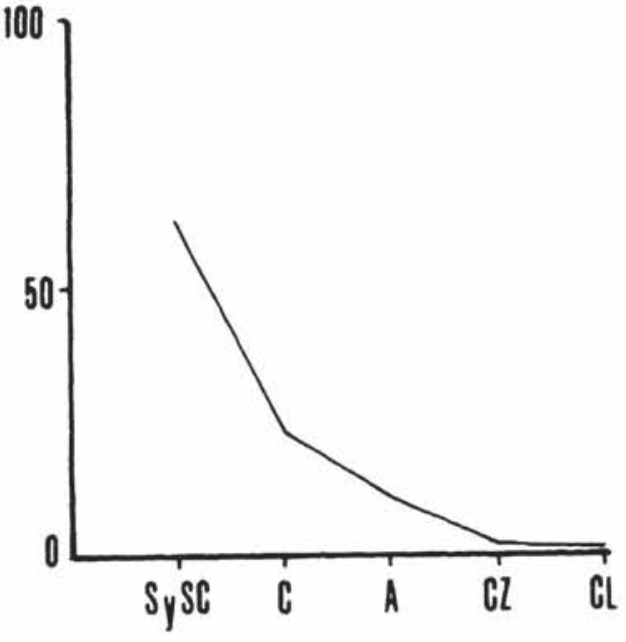

Figura 4B

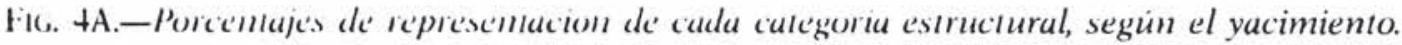
B.-Porcentajes de representación de cada materia prima en los yacimientos (siglas en Tabla III).

TABLA III

Distribución y representación de las materias primas por categorias estructurales. Las cifras en negrita son los efectivos reales. A su derecha, los porcentajes de las materias primas, respecto a las categorias estructurales. S: Sílex errático; SC: Sílex de cueva; C: Cuarcita; A: Arenisca; CZ: Cuarzo; CL: Caliza y T.: Total

\begin{tabular}{|c|c|c|c|c|c|c|c|}
\hline & $\mathbf{s}$ & sc & c & A & cz & CL & $\mathbf{T}$ \\
\hline B & & & $70 / 85.3$ & $10 / 12.2$ & & $2 / 2.4$ & 82 \\
\hline BP & $177 / 65.3$ & $14 / 5.1$ & $44 / 16.2$ & $26 / 9.6$ & $4 / 1.4$ & $6 / 2.2$ & 271 \\
\hline BN1G & $30 / 44.1$ & $2 / 2.9$ & $21 / 30.8$ & $10 / 14.7$ & $4 / 5.8$ & $1 / 1.4$ & 68 \\
\hline BN2G & $68 / 53.1$ & $11 / 8.6$ & $26 / 20.3$ & $22 / 17.1$ & & $1 / 0.7$ & 128 \\
\hline IND. & 133 & & 3 & 7 & 6 & 3 & 152 \\
\hline $\mathbf{T}$ & $408 / 58.2$ & $27 / 3.8$ & $164 / 23.4$ & $75 / 10.7$ & $14 / 2$ & $13 / 1.8$ & 701 \\
\hline
\end{tabular}


Contamos con un total de 50 BNIG para TD y TG-TN, más 8 fragmentos de los que sólo puede determinarse la materia prima y otras 5 piezas en las que existen dudas sobre su posible origen natural. Se trata fundamentalmente de cantos de cuarcita y arenisca, indudablemente transportados por el hombre, aunque posiblemente no modificados por él, sino por otros agentes postdeposicionales. Existen además 5 piezas que realmente son BN2G, aunque talladas y explotadas como si fueran BN1G, sin presentar caracteres - retoque, por ejemplo- que lleven a incluirlas entre los instrumentos. Así, pues, las estudiaremos como si fueran BN1G.

De las 55 piezas que estudiamos de TD y TG-TN, 38 pueden ser consideradas claramente como BN1G neutras (núcleos). Esto no excluye que algunas de ellas presenten un segmento retocado, debido probablemente a su utilización en una función más directa, una vez finalizada la secuencia de talla. Las otras 17 BNIG han sido consideradas como BN1G no neutras, entre las que se ha incluido las piezas de difícil caracterización. En este trabajo sólo se estudiarán las BN1G neutras; es decir, aquéllas cuya función primaria es la producción de BP (lascas). Entiéndase que este análisis se efectúa sobre la pieza y que ésta presenta sólo la secuencia de talla inmediatamente anterior a su abandono. La presencia regular de un tipo de BN1G y la morfología y caracteres técnicos de la BP producto permiten inferir la existencia de un tipo de talla "estandar", repetida al menos durante gran parte de la secuencia.

\section{ANALISIS DE BN1G}

Cinco aspectos permiten analizar, a nuestro juicio, el tipo de talla llevado a cabo sobre una BN1G (Mosquera, 1989): la disposición de los levantamientos, la configuración de los puntos de impacto, el número y disposición relativa de las plataformas de percusión, la inclinación de las extracciones en la superficie de lascado respecto a la plataforma de percusión y las morfologías finales. La ordenación supeditada de los cuatro primeros factores conlleva una morfología final específica. Son, por tanto, aspectos fundamentales para el análisis, por lo que pasamos a explicarlos a continuación.

La disposición de los levantamientos se refiere a la posición relativa de éstos durante la secuencia de talla. Existen dos alternativas: disposición no adyacente (ver Fig. 6D) y disposición adyacente (Figs. 9-13).

Esta última, a su vez, puede presentarse como disposición adyacente respecto a las caras de las lascas (Fig. 5A) o respecto a sus aristas (Fig. 5B). Como puede observarse en las mencionadas figuras, la diferencia entre ambos modelos reside en que en el primer caso cada BP se ha de situar detrás de la anterior (talla en Volumen) -como es el caso de la talla en "tranches" (Brézillon, 1968) - y en el segundo, cada pieza se ha de situar al lado de la anterior (talla Marginal). En este último caso se trata de una forma de talla en la cual se levanta la periferia de la BN1G.

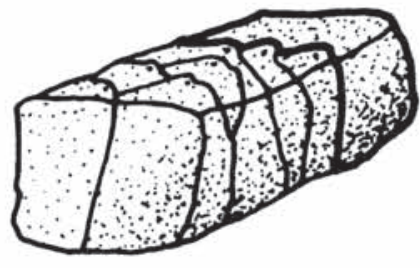

A

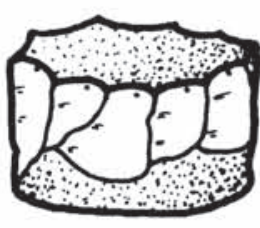

$\mathbf{B}$

FIG. 5.-Disposición adyacente de los levantamientos: $A$, respecto a las caras de la lasca $(B P) ; B$, respecto a las aristas de la lasca (BP). 
La configuración de los puntos de impacto puede ser rectilínea (Fig. 6A), en espiral (Fig. 6B), alterna (Fig. 6C) o no manifiesta (Fig. 6D).

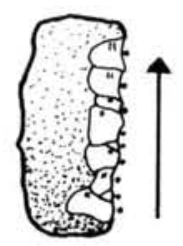

A

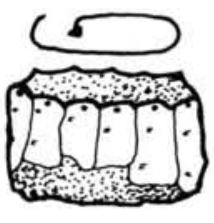

B

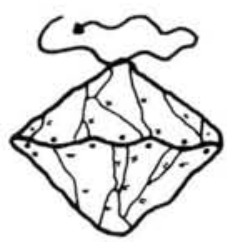

C

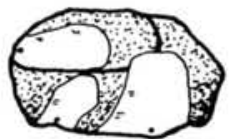

D

Fig. 6.-Con/iguracion de los puntos de impacto: $A$, rectilinea; $B$, en espiral o circular; $C$, alterna; $D$, no manifiesta.

Durante la talla de una BN1G pueden utilizarse una, dos o más plataformas de percusión. En un caso típico como el representado en la figura $6 \mathrm{C}$ consideramos que se han utilizado dos plataformas de percusión contiguas, dispuestas para la talla de modo opuesto. Esta figura representaría una talla Marginal y bifacial.

La inclinación de las extracciones se refiere al ángulo entre plataforma de percusión y superficie de lascado. Se proponen los modos Plano (0-30 grados), Simple (30-60 grados) y Abrupto (60-90 grados), representados por la figura 7A, 7B y 7C, respectivamente.

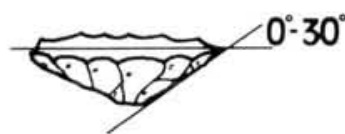

A

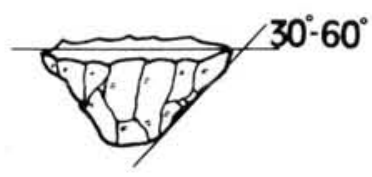

B

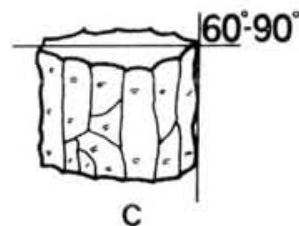

C

Fig. 7.-Inclinacion de las exraciones: A, planas; B, simples; C; abruples.

Como ya se ha mencionado, la morfología final específica de cada núcleo depende de las diferentes combinaciones que se realicen con estos cuatro factores. De esta forma, cualquier tipo de BN1G puede ser explicada, incluso aquéllas de las que no conocemos su finalidad última. Un chopping-tool, por ejemplo, puede ser técnicamente descrito (Figs. 10B y D; 12B y D) como una BN1G (canto en este caso) sometido a una talla Marginal, con configuración alterna de los puntos de impacto, con dos plataformas de percusión contiguas y dispuestas para la talla de modo opuesto y con negativos Planos o Simples respecto a la plataforma de percusión.

Las combinaciones más evidentes y comunes se explican a continuación.

A. Disposición No-Adyacente de los levantamientos. Son levantamientos independientes unos de otros (Fig. 6D).

T. P., 1992, no 49 


\section{B. Disposición adyacente}

a) Talla en Volumen: Cada levantamiento se sitúa detrás del previo. La configuración de los puntos de impacto puede ser rectilínea (Fig. 8) y alterna (Fig. 9). Esta última con una plataforma de percusión ha sido identificada por los autores de este trabajo en el yacimiento del Pleistoceno Medio de Aridos (Madrid).

b) Talla Marginal: cada levantamiento se sitúa al lado del previo. La configuración de los puntos de impacto puede ser rectilínea (Fig. 10), espiral (Fig. 11) o alterna (Fig. 12).

Las morfologías que incorporan una plataforma de percusión usualmente modifican sólo una cara; son los unifaciales. Aquellas con dos plataformas de percusión modifican normalmente dos caras y son los bifaciales. La configuración rectilínea de los puntos de impacto (Fig. 10) presenta morfologías muy conocidas; e.g. instrumentos sobre canto unifaciales y bifaciales y morfologías ortogonales de núcleos.

La configuración en espiral de los puntos de impacto (Fig. 11) puede verse en unifaciales planos (Fig. 11A), discoides, bifaces y bifaciales planos (Fig. 11B), núcleos piramidales (Fig. 11C), núcleos bipiramidales (Fig. 11D), núcleos prismáticos (Fig. 11E y 11F) y núcleos poliédricos (Fig. 11F).

La configuración alterna de los puntos de impacto (Fig. 12) produce morfologías similares a las representadas en la figura 11 .

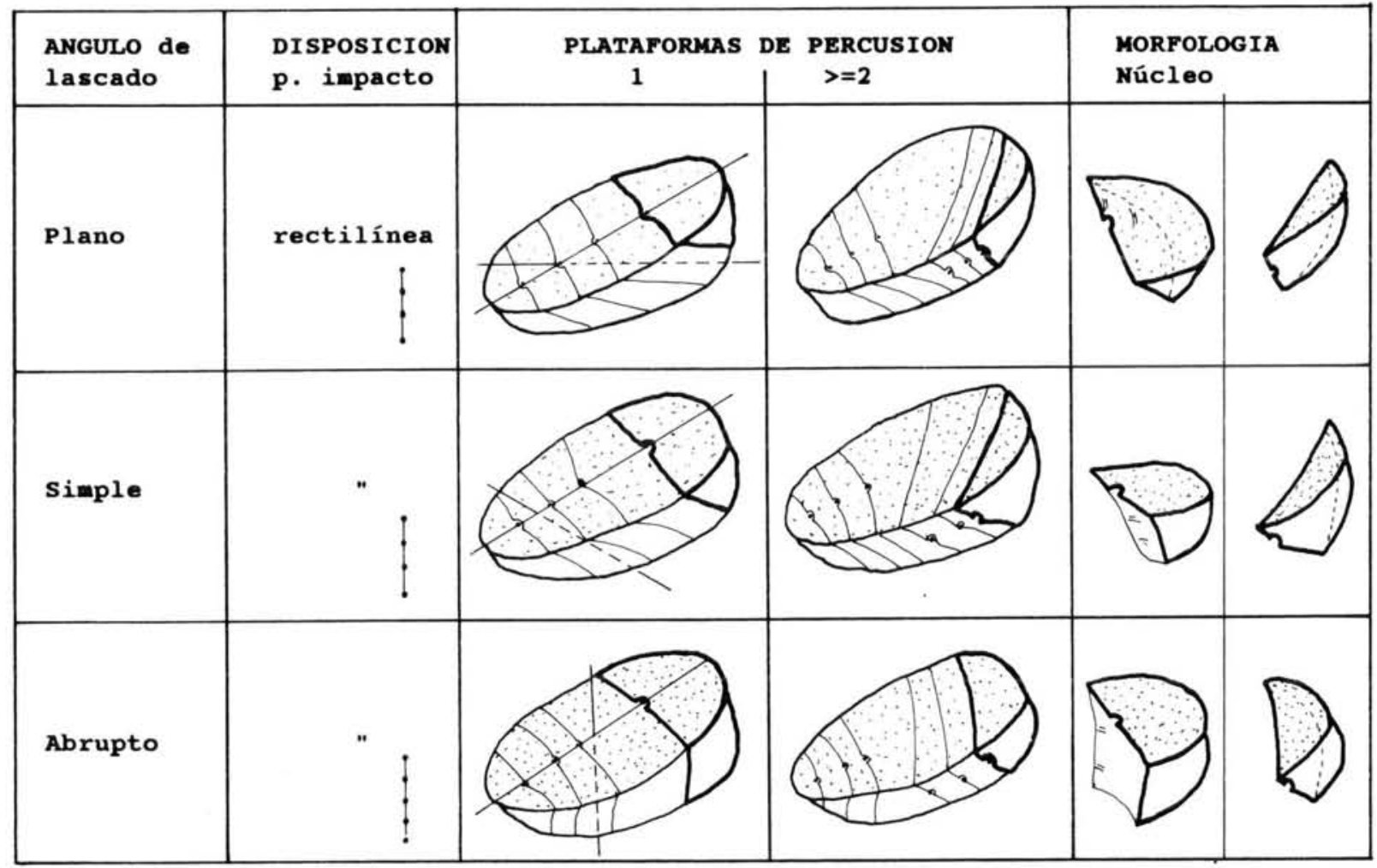

Fig. 8.-Disposición Adyacente de los levantamientos: Talla en Volumen. Configuración rectilínea de los puntos de impacto. 


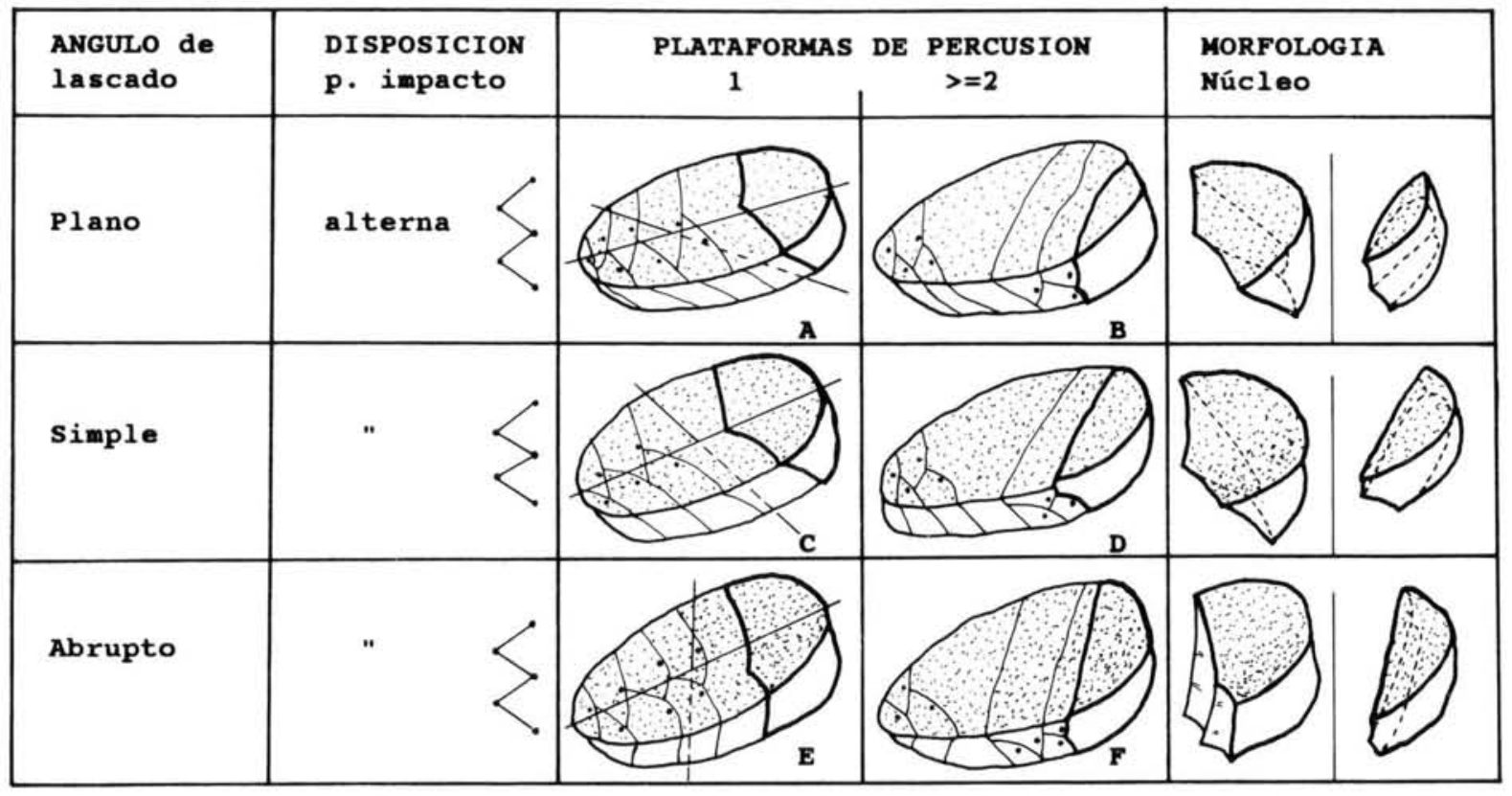

Fig. 9.-Disposicion Advacente de los kevantamientos: Ialla en Volumen. Configuracion alterna de los puntos de impacto.

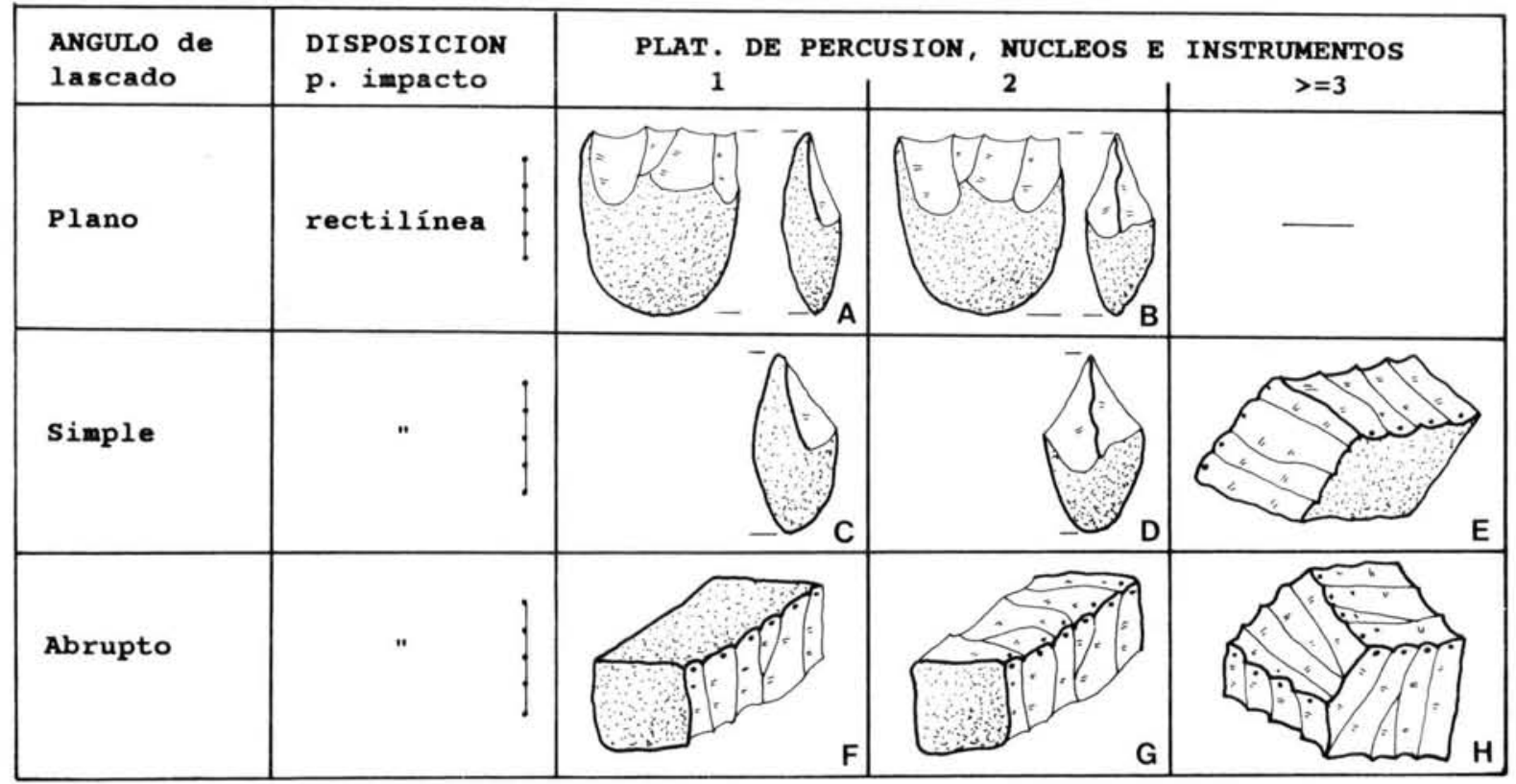

Fig. 10.-Disposicion Adyacente de los levantanientos: Talla Marginal. Con/iguracion rectilinca de los puntos de impacto.

T. P., 1992, nº 49 


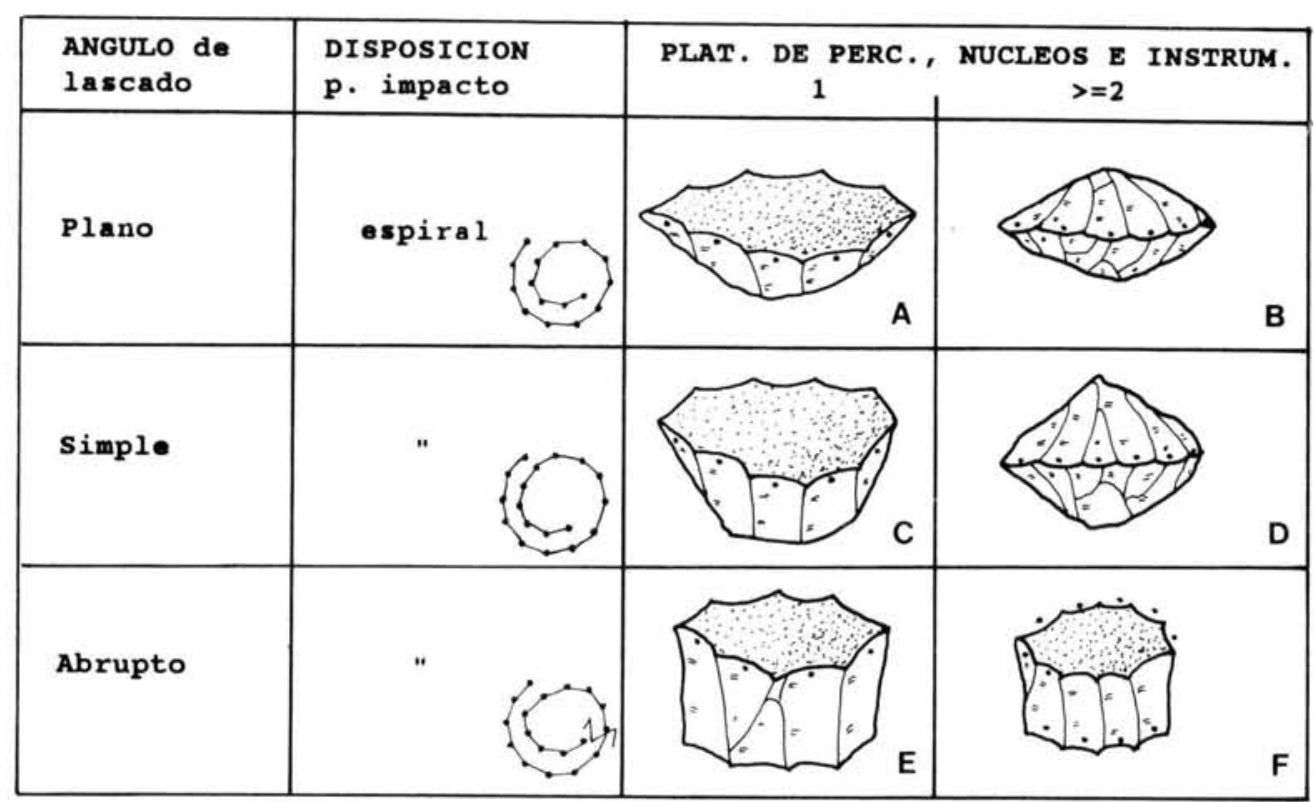

Fig. 11.-Disposición Adyacente de los levantamientos: Talla Marginal. Configuración espiral de los puntos de impacto.

\begin{tabular}{|c|c|c|c|}
\hline $\begin{array}{l}\text { ANGULO de } \\
\text { lascado }\end{array}$ & $\begin{array}{l}\text { DISPOSICION } \\
\text { p. impacto }\end{array}$ & $\begin{array}{c}\text { PLAT. DE PERC. , } \\
1\end{array}$ & $\underset{>=2}{\operatorname{NUCLEOS} E}$ INSTRUM. \\
\hline Plano & alterna & & \\
\hline Simple & $"$ & & \\
\hline Abrupto & $"$ & & \\
\hline & & E & $\mathrm{F}$ \\
\hline
\end{tabular}

FIG. 12.-Disposición Adyacente de los levantamientos: Talla Marginal. Configuración alterna de los puntos de impacto. 
c) Disposición Mixta de los levantamientos. Se refiere a las secuencias de talla en las que están presentes diferentes patrones de levantamientos. Así, por ejemplo, una secuencia de talla Marginal en la que la disposición de los puntos de impacto es rectilínea en un primer momento y en espiral al final.

d) Otros. Incluye otros patrones en los que se da la talla Marginal. Por ejemplo, los esferoides y núcleos poliédricos en los que un levantamiento ha sido utilizado como plataforma de percusión del siguiente. La configuración de los puntos de impacto posiblemente será diferente en cada caso.

c. Levantamientos Aislados. Pertenecen a este grupo dos tipos de piezas: aquéllas con un único levantamiento, cuyo origen natural o antrópico es difícil de evaluar, y aquellas piezas modificadas que muestran un patrón generalizado de talla, excepto para unos pocos levantamientos. Técnicas de talla como la Levallois y la Kombewa se sitúan en este grupo. La técnica Levallois (Fig. 13) podría ser descrita como un método de talla Marginal centrípeto y bifacial con inclinación Plana de los levantamientos en una cara y Simple o Abrupta en la otra. La configuración de los puntos de impacto es espiral. Cuando el núcleo está preparado - núcleo en caparazón de tortuga- el patrón de talla cambia y algunas lascas son levantadas según el patrón de talla en Volumen -cada levantamiento se sitúa detrás del previo-, normalmente con una configuración alterna de los puntos de impacto. Estos últimos levantamientos son las llamadas lascas Levallois y representan un cambio en el patrón de talla utilizado; esto es, son levantamientos aislados respecto al patrón generalizado en el resto de la secuencia. Por ello, consideramos estas técnicas como casos específicos de determinados métodos de talla.

\section{LAS BN1G DE ATAPUERCA}

Al menos el $70 \%$ de la BN1G neutras recuperadas presentan una disposición adyacente de los levantamientos respecto a las aristas de las BP; es decir, una talla Marginal. Este tipo de talla se ha podido identificar repetidamente en los niveles excavados, que son los superiores en el conjunto de los rellenos cársticos. A continuación se expondrán las características de estas piezas, atendiendo a su pertenencia a los conjuntos de niveles previamente especificados.
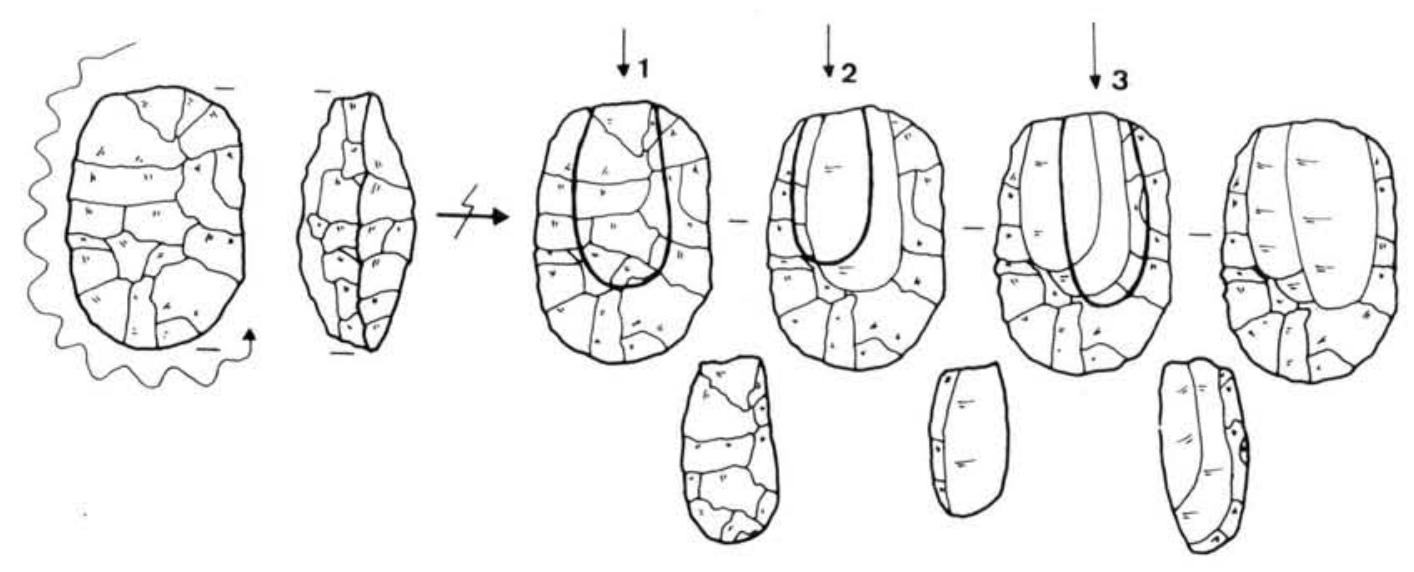

FIG. 13.-Levantamientos aislados respecto al patrón de talla utilizado en el resto de la secuencia: técnica Levallois.

T. P., 1992, $\mathrm{n}^{\circ} 49$ 
1. Conjunto de niveles superiores (TG-TN/TD). De las 47 BN1G que forman parte de estos conjuntos, 32 son claramente neutras, mientras que otras 15 presentan problemas de asignación. El $87,5 \%$ de las BN1G neutras son bifaciales y el 12,5\% restante, unifaciales. Ambos grupos participan de un índice muy alto de talla Marginal, asegurado en 26 piezas $(81,2 \%)$ y dudoso en otras $6(18,7$ 9). No se ha identificado ningún ejemplar claro de talla en Volumen.

Un hecho a destacar es la alta presencia entre los bifaciales con talla marginal de una cara con levantamientos Planos, mientras que la otra los tiene Simples (Lám. III. A.). Esto ocurre en 20 de 24 piezas; las otras 4 presentan en ambas caras levantamientos Simples. En general, la cara de levantamientos Planos refleja, o bien negativos con configuración circular de los puntos de impacto, o bien negativos con configuración rectilinea. En este último caso, se trata de una doble hilera opuesta de levantamientos Planos y rectilíneos. Los negativos de la cara opuesta -Simples con tendencia Abrupta - presentan una orientación convergente. Aparentemente, la cara plana parece haberse utilizado como plataforma de percusión preparada y la inclinada como superficie de lascado, por lo que la configuración de los puntos de impacto de las caras Simples puede ser circular con alternancias puntuales con la otra cara, con el fin de regularizar la plataforma de percusión. Es usual que la cara de levantamientos Planos contenga menor número de negativos que la cara opuesta.

Otro hecho a destacar es que 7 de las BN1G bifaciales presentan un engrosamiento distal natural o utilizado en ocasiones como tercera plataforma de percusión. Esto genera morfologías finales muy similares a las documentadas por algunas BN2G (lascas retocadas). Las semejanzas entre las categorías estructurales serán comentadas más adelante. Dentro de este gran subconjunto de BN1G con talla Marginal y bifacial, existen algunas piezas que responden a las características generales comentadas, pero presentan una asimetria sagital muy fuerte debido al desajuste espacial entre las dos caras.

Finalmente, existen 6 BN1G - correspondientes también a los niveles superiores- que no responden con suficiente claridad a los modelos presentados. Probablemente se trate de talla Marginal. Suelen reflejar la utilización de dos plataformas de percusión contiguas pero no opuestas, lo que deriva en la formación de dos aristas sagitales y una morfología final cúbica. Todo ello, unido al escaso número de levantamientos, hace pensar que son fragmentos de otras BN1G de mayores dimensiones, aprovechados tras la fractura.

Las morfologías de las BN1G correspondientes al más generalizado tipo de talla -Marginal, centrípeto y bifacial - son multiangulares y circulares para la vista horizontal y bicónicas o cónicas para la morfología sagital y transversal. La forma horizontal suele presentar cuatro o cinco ángulos para las piezas de tamaño medio y grande (más de $60 \mathrm{~mm}$.) y de más de cinco ángulos - consideradas como circulares- para las menores. La media de sus dimensiones es de $61,59 \times 4,05 \times 32,61$ $\mathrm{mm}$. Las características técnicas de la única BN1G correspondiente al Conjunto de niveles mediosuperiores (TG-TN) no difiere de las vistas hasta el momento.

2. Conjunto de niveles medio-inferiores. Existe otro tipo de BN1G neutras aparecidas en los niveles medios-inferiores del relleno de TD. Se trata de 4 piezas. Dos de ellas, de grandes dimensiones $(200 \times 150 \times 100 \mathrm{~mm}$., aprox.), son de sílex errático, presentan una morfología cúbica y no reflejan unas pautas organizadas de extracción de BP, por lo que en origen pudieron ser talladas repetidamente según una disposición no adyacente de los levantamientos. Al margen de ello, muy bien puede tratarse de una reducción de masa correspondiente a las primeras fases de la talla Marginal, más generalizada al menos en los niveles superiores (Lám. III. C). Las otras 2 piezas, ambas de cuarcita, están parcialmente talladas. El tipo de talla que presentan es Marginal y la orientación de los levantamientos es ligeramente convergente, lo que indica un modelo de talla similar al de los niveles superiores. La dimensión media del conjunto es de 150,79 × 107,59 × 70,41 mm.

3. Conjunto de niveles inferiores. En contraposición al gran conjunto de talla Marginal, sólo una BN1G - correspondiente a los niveles inferiores de TD, por encima del límite Matuyama/Bruhnes- 
presenta una disposición advacente en los levantamientos respecto a las caras de la BP (Talla en Volumen). Se trata de una gran BN1G de cuarcita $(114 \times 77 \times 102 \mathrm{~mm}$.) sobre la que se efectuaron seis levantamientos, cada uno detrás del anterior, desde una sola plataforma de percusión. Los dos primeros atravesaron todo el espesor de la pieza $(102 \mathrm{~mm}$.), mientras que los cuatro restantes quedaron reflejados, lo que motivó la formación de una superficie de lascado convexa, la consiguiente dificultad de tallarla y, finalmente, el abandono de la pieza (Lám. IV. A). Otras 2 BNIG neutras forman parte de este conjunto, unifacial en su totalidad, y compuesto exclusivamente por piezas de cuarcita. Ambas presentan escasos levantamientos, a veces discontinuos. La dimensión media del conjunto es de $99,33 \times 69 \times 55,33 \mathrm{~mm}$.

\section{LAS BP Y BN2G DE ATAPUERCA}

Es obvio que el tipo de talla repercute directamente sobre las características de las Bases Positivas que se produzcan. Por ello, pretendemos contrastar el modelo de talla reconocido en las BN1G de Atapuerca, con los rasgos técnicos de las BP y BN2G recuperadas. De modo general, las BP y BN2G contienen información de una serie de caracteres cuyo análisis permite su sistematización: forma de la cara ventral; cantidad de córtex del talón, forma horizontal y frontal del mismo, facetaje y tipo; cantidad de córtex, forma sagital, horizontal y transversal de la cara dorsal, así como el número de inclinación de los levantamientos previos a la extracción; ángulo de lascado, etc. (Carbonell, 1987).

Dentro del análisis de estos caracteres, varios de ellos y su asociación resultan, a nuestro juicio, de mayor importancia para el tema tratado. Estos son el tipo de cara talonar y facetaje; la forma frontal de esta misma cara, el número y diseño de las nervaduras dorsales y la morfología horizontal de la pieza; por último, la orientación de los levantamientos previos a la extracción de la lasca.

En Atapuerca, la cara talonar es de tipo plataforma, generalmente de gran tamaño, excepto en un pequeño conjunto de piezas con talones pequeños (10 mm. aprox.) de morfología oval. En el 69 \% de los casos, el ángulo entre plataforma y bulbo oscila entre 100 y 120 grados, dominando ligeramente el grupo de efectivos con ángulos entre 109 y 120 grados (Martín Nájera, 1990) (6). Las BP y BN2G de grandes plataformas talonares se caracterizan especialmente por dos aspectos: en primer lugar, la amplitud de las facetas y la existencia de ángulos relativamente agudos entre ellas, lo cual induce a confundir facetas de la plataforma talonar con lados de la pieza; en segundo lugar, presentan una fuerte nervadura en la delimitación entre cara talonar y cara dorsal. Nervadura y facetas corresponden a una parte de la arista sagital (nervadura) de la BN1G bifacial y a pequeñas áreas de la plataforma de percusión (facetas) adyacentes a dicha arista levantado todo con la extracción de la Base Positiva.

El tamaño de estas plataformas talonares es tal que su amplitud suele coincidir con el mayor espesor de la pieza. De hecho, muchas de ellas presentan series de retoques en la nervadura cara talonar-cara dorsal, realizados posiblemente con el fin de adelgazar a la pieza.

Las piezas se distribuyen en tres subgrupos - coincidentes a grandes rasgos con los grupos de tamaño- atendiendo a las morfologías, que en general son de tipo trapezoidal (38\%), cuadrangular $(26 \%)$ y triangular $(32,6 \%)$ :

1. BP y $B N 2 G$ de pequeño formato (hasta $30 \mathrm{~mm}$.). A este grupo pertenecen el $36 \%$ de las piezas recuperadas en ambos yacimientos. La mayor parte de ellas presentan morfologías trapezoidales, con una o ninguna nervadura dorsal y con fuertes charnelas. Muchas de ellas pueden identificarse como BP-esquirlas de talla, aunque algunas fueron seleccionadas para ser retocadas.

(6) A. Martín Nájera es Directora del Museo Pérez Comendador-Leroux de Hervás (Cáceres) y del Museo Etnográfico Textil Provincial de Plasencia (Cáceres). Forma parte del equipo de Atapuerca desde 1980. Realizó su Tesis de Licenciatura (1986) sobre el análisis técnico de la industria lítica de los yacimientos de la Sierra de Atapuerca.

T. P., $1992, \mathrm{n}^{2} 49$ 
2. BP y BN2G de formato medio (de 30 a $60 \mathrm{~mm}$.) (Lám. III. B). constituyen el $43,7 \%$ de los efectivos y presentan varias morfologias:

a) morfología trapezoidal que, como en el caso anterior, pueden tener una o ninguna nervadura dorsal. Algunas de ellas fueron también seleccionadas para ser retocadas.

b) morfología cuadrangular, asociada a caras dorsales que presentan nervaduras cruzadas. Estas piezas son el producto de levantar una superficie plana de la BN1G, tallada previamente de modo Marginal centrípeto.

Otro tipo de BP y BN2G tiene formatos igualmente cuadrangulares, pero los negativos que presentan en sus caras dorsales son subparalelos. Pueden tener dos o tres nervaduras. Estas, junto con las formas triangulares - con una nervadura longitudinal, a veces desviada - son las piezas resultantes de la extracción sistemática de BP de la superficie de lascado (cara de la BN1G con levantamientos Simples y Abruptos). La única diferencia entre ellas es que las triangulares levantaron una porción de BN1G con una nervadura, mientras que la porción de las cuadrangulares incluía varias. Así, pues la morfología de la lasca depende de la cantidad y disposición de las nervaduras que levante.

3. BP y BN2G de gran formato (más de $60 \mathrm{~mm}$.; generalmente, más de $80 \mathrm{~mm}$.) (Lám. IV. B). A este grupo pertenece el 20,2\% de los efectivos. Como las otras, presentan morfologías trapezoidales y triangulares, aunque en muchos casos no se aprecia la forma original debido a la fuerte modificación que sufrieron tras su extracción. En general, son más anchas que largas y poseen una nervadura que cruza la pieza en diagonal en las de menor espesor (Lám. IV. C.2) y a lo largo del eje longitudinal de la pieza en las más gruesas (Lám. IV. C.1). Estas últimas son las utilizadas para la manufactura de grandes útiles como bifaces, hendedores,... Su plataforma talonar continúa siendo bifacetada o multifacetada, lo cual indica preparación previa a su extracción. Este tipo de BP proviene posiblemente de los primeros levantamientos efectuados sobre el nódulo. El hecho de que no haya aparecido en los niveles superiores ninguna BN1G de una dimensión suficiente como para presentar negativos de estas grandes BP y la presencia de dos grandes BN1G globulares en los niveles medios, nos lleva a suponer que ambos tipos de núcleos pueden corresponder al mismo "estandar" de talla, pero a diferentes etapas de la secuencia; así, los núcleos de los niveles más modernos serían trabajados posteriormente mediante la explotación centrípeta bifacial documentada tan ampliamente.

\section{RESUMEN Y CONCLUSIONES}

Hemos visto que la talla lítica en Atapuerca puede modelizarse de la siguiente manera. La forma de talla normalmente es Marginal bifacial con levantamientos Planos, Simples y Abruptos, mediante la cual se producen varios tipos de BP, la mayoría cortas, procedentes de la explotación de la superficie de lascado con levantamientos Simples, y otras que resultan del levantamiento de las caras opuestas, con extracciones Planas. Una de las características más significativas de las BP producto de la talla Marginal es su morfología trapezoidal y triangular. Las primeras pueden no tener nervaduras dorsales y presentar fuertes charnelas, o tener una o dos nervaduras de dirección longitudinal o desviada respecto al eje de la pieza. Las segundas suelen presentar una arista longitudinal. Ambos tipos documentan una orientación subparalela en los negativos de sus caras dorsales. Por su parte, las BP producto de levantar la cara de la BN1G con extracciones centrípetas Planas, presentan tres o más nervaduras cruzadas y una morfología cuadrangular-circular. Por último existe una serie de grandes BP (la mayoría aprovechadas para la manufactura de grandes útiles como bifaces, hendedores, etc.), también de morfologia trapezoidal o triangular, cortas y anchas y con una arista dorsal muy marcada - desviada o no- que actúa siempre como eje 
longitudinal de la pieza a retocar. Este último grupo, junto con grandes fragmentos de regularización de plataformas de percusión, se cree provienen de los primeros levantamientos efectuados sobre grandes bloques de silex errático. Estos levantamientos aprovecharian las aristas abruptas que naturalmente presentan estos bloques y corresponderían a las primeras fases de la secuencia de explotación periférica, manifiesta en los yacimientos de Atapuerca.

En general, existe una presencia importante (67\%) de transformación en Bases Negativas de Segunda Generación de las BP con una o más nervaduras en sus caras dorsales.

Por otra parte, hemos considerado otra serie de puntos a tener en cuenta para la caracterización general de la explotación en estos yacimientos:

1. Polaridad. Se documenta mayoritariamente una polaridad convergente (orientación de los levantamientos hacia un punto) en los niveles superiores. El muestreo sobre los niveles mediosinferiores del relleno de TD ha procurado la obtención de dos grandes BN1G que no presentan una polaridad manifiesta o unívoca, mientras que otras dos reflejan al menos una tendencia convergente. Por último, un único ejemplar documenta polaridad longitudinal y talla en volumen, y pertenece a los niveles inferiores, más antiguos, del relleno de TD.

2. Transversalidad. Se puede observar que sobre el conjunto industrial de Atapuerca domina el concepto de transversalidad. Esto es, la mayoría de los objetos poseen formas no apuntadas, excepto en el caso de algunas BN2G (bifaces, por ejemplo). El resto - BP, BN2G e incluso BN1G neutras retocadas- participan del hecho de poseer morfologías cuadrangulares, pentagonales, etc. Esto estaria en consonancia con la funcionalidad de las ocupaciones, dirigida supuestamente a la manipulación de alimentos dentro de las cavidades.

3. Existe otra característica en la industria de Atapuerca y es su gran homogeneidad, visible en el carácter de transversalidad generalizado, en el tipo de talla sistemáticamente reiterado y, sobre todo, en la similitud entre las categorías estructurales elaboradas. Ejemplo de ello es que incluso el aspecto global de varias BN2G (5 piezas) se confunde con el de las BN1G, debido a la utilización en ambas de métodos de talla, configuración y modificación, similares.

4. El grado de aprovechamiento de la materia prima en Atapuerca parece haber sido muy fuerte. Por ejemplo, no existe una selección de BP por tamaños para ser transformadas en BN2G. Existen muestras de esta última categoría desde $15 \mathrm{~mm}$. hasta los grandes útiles sobre lasca (entre 130 y $50 \mathrm{~mm}$. de longitud). Como aproximación, pensamos que el grado de aprovechamiento puede deducirse por la apreciación conjunta del carácter de facialidad del núcleo (unifacial, bifacial, multifacial), unido a la cantidad de superficie modificada en el mismo y unido, igualmente, a la diferencia de tamaño entre los últimos negativos de una BN1G y el de los levantamientos que podrian seguir obteniéndose.

De esto modo, observamos un grado de aprovechamiento muy fuerte para el Conjunto de niveles superiores, ya que la mayoría de las BN1G neutras son bifaciales (289 sobre 32); además 20 mantienen un grado de explotación muy alto, por presentar toda la superficie modificada y una dimensión media de los núcleos de $50,69 \times 38,21 \times 28,27 \mathrm{~mm}$.; 8 piezas sufrieron una modificación media y sólo 4 están escasamente explotadas, ya que el tamaño de los negativos que muestran sus caras de lascado es menor que el de las BP que puedan potencialmente obtenerse.

Pese al reducido número de efectivos, en los Conjuntos de niveles medio-inferiores (4 piezas) e inferiores ( 3 piezas) -representados exclusivamente en TD-, es significativo que ambos grupos posean un tamaño medio de sus BN1G neutras mucho mayor que el obtenido para el Conjunto de niveles superiores. Para el Conjunto medio-inferior esta media es de $150,79 \times 107,59 \times 70,41 \mathrm{~mm}$. De las 4 piezas, 2 son bifaciales y 2 unifaciales, con lo que la modificación de la superficie disminuye en relación a los niveles superiores. Además, todas pueden ser consideradas como escasamente explotadas, pues el tamaño medio de los negativos está muy por debajo del potencial 
del núcleo abandonado. Algo similar ocurre con el Conjunto de niveles inferiores de TD, en el que el tamaño medio es de $99,33 \times 69 \times 55,33$, los 3 efectos son unifaciales y se presentan poco explotados.

Un aspecto importante respecto a las diferencias entre los conjuntos es la utilización aparentemente selectiva de la materia prima. Aunque sujeto al posible sesgo provocado por la escasez de datos actual, no deja de ser significativo que la cuarcita, utilizada en un $26 \%$ de las BN1G de los niveles superiores de TD y TG-TN, en los medios e inferiores adquiere una significación del 71,4\%. Igualmente interesante es que sólo 3 de las 10 BN1G realizadas sobre arenisca puedan ser identificadas como BN1G neutras. Parece que la utilización de este material en la producción de BN1G iría encaminada a la obtención de instrumentos, más que a la de núcleos.

De todos los caracteres comentados a lo largo de este trabajo, varios aspectos tecnológicos inducen a encuadrar la industria de los niveles superiores en la cadena operativa achelense: presencia constante de bifaciales totalmente modificados, aristas frontales irregulares y sinuosas, asimetria sagital, presencia de útiles muy gruesos, producción de grandes BP y reiteración de morfologías trapezoidales y cuadrangulares.

No obstante, existe otra serie de caracteres técnicos muy significativos a la hora de concretar el proceso evolutivo tecnológico: utilización regular de método de talla bifacial Marginal y en espiral que confiere a los núcleos su aspecto cónico; presencia de una orientación de los negativos mayoritariamente convergente provocada por la talla Marginal centrípeta; abundante presencia de morfologías multiangulares con tendencia a la circularidad en las BN1G neutras y, por último, una gran homogeneidad de todas las categorías estructurales elaboradas. Todos estos rasgos serán característicos de las industrias del Pleistoceno superior inicial.

Por todo ello, y en consonancia con el estudio global de la industria (Carbonell et alii, 1987c), el proceso de explotación lítico reflejado hasta el momento en los niveles superiores de los rellenos de Atapuerca, debe encuadrarse en un momento final de la cadena operativa achelense.

\section{AGRADECIMIENTOS}

Los autores firmantes deseamos expresar nuestro agradecimiento al Prof. Emiliano Aguirre por la ayuda que nos ha prestado en todo momento, indispensable para el conocimiento de los contextos geológico, cronológico y paleontológico de los yacimientos. Este trabajo ha sido subvencionado por el Proyecto Atapuerca, № PB90.0126.603.01 de la DGCYT y en parte (M. M.) por una beca de Formación de Personal Investigador de la Comunidad de Madrid, a través de su Plan Regional de Investigación.

\section{BIBLIOGRAFIA}

Aguirre, E., Bermúdez de Castro, J. M. y Carbonell, E. (eds.) (1987): El Hombre fósil de Ibeas y el Pleistoceno de la Sierra de Atapuerca I. Monografía. Consejería de Cultura y Bienestar Social. Junta de Castilla-León, Valladolid. 439 pp.

BERmúdeZ DE CASTRO, J. M. (1990): «Los yacimientos mesopleistocenos de la Sierra de Atapuerca: resultados (1978/1990) y expectativas". En J. Barrio (Coord.) Ciencias y Técnicas al Servicio de la Investigación Arqueológica. Fundación F. Giner de los Ríos. Institución Libre de Enseñanza, Madrid: 1-26.

Brézillon, M. (1968): La Dénomination des objets de pierre taillée. Centre National de la Recherche Scientifique. Bordeaux, 423 pp.

Carbonell, E. (1987): «Human Development in the Framework of Lithic Operative Chains». En Sistemes d'análisi en Prehistória. C.R.P.E.S., Girona: 68-82.

Carbonell, E., Guilbaud, M. y Mora, R. (1983): "Utilización de la Lógica-Analítica para el estudio de Tecnocomplejos a cantos tallados". Cahier Noir, 1: 1-64. C.R.P.E.S., Girona.

Carbonell, E., DíEZ Fernández-Lomana, C. y Soto, E. (1987a): «Estudio preliminar de los Suelos de Ocupación de Atapuerca (Burgos)n. En E. Aguirre et alii (eds.) (1987): 425-439.

T. P., 1992, $\mathrm{n}^{2} 49$ 
Carbonell, E., Dízz Fernández-Lomana, C. y Martín Nájera, A. (1987b): "Análisis de la industria lítica del Complejo de Atapuerca (Burgos)». En E. Aguirre et alii (eds.) (1987): 389-425.

Carbonell, E., Mosquera, M., Ollé, A., Sala, R., Vaquero, M. y Vergés, J. M. (1992): «New elements of the Logical Analytic System». Cahier noir, 6, 61 pp.

Carracedo, J. C., Heller, F., Soler, V. y Aguirre, E. (1987): «Estratigrafía magnética del yacimiento de Atapuerca: determinación del límite Matuyama/Bruhnes. En E. Aguirre et alii (eds.) (1987): 193-199.

GRUN, R. y AGUiRRE, E. (1987): "Datación por "ERS" y por la serie del "U" en los depósitos cársticos de Atapuerca». En E. Aguirre et alii (eds.) (1987): 201-205.

Martín-Nájera, A. (1990): "El Complejo Litico de la Trinchera de Atapuerca (Burgos-España). Excavaciones 1980 1986. E. Carbonell y E. Aguirre (eds.) Univ. de Tarragona - Museo Nac. de Ciencias Naturales. 238 pp.

Mosouera, Martinez, M. (1989): Análisis y secuencias de Talla litica sobre sílex. Aportación experimental a los métodos de talla en volumen y marginal centripeto. Tesis de Licenciatura. Dpto. Prehistoria. U.C.M., Madrid, 284 pp. (inédita).

Rodríguez, X. P. (1991): El Complejo mesolpleistocénico de la Sierra de Atapuerca (Burgos): Variabilidad técnica de la industria litica de Trinchera Dolina. Tesis de Licenciatura. Univ. de Tarragona, 400 pp. (inédita).

RosAS, A. (1987): "Two new mandibular fragments from Atapuerca/Ibeas (SH site). A reassessment of the affinities if the Ibeas mandibles samplew. Journal of Human Evolution, 16: 417-427.

Rosas, A., Bermúdez de CASTro, J. M. y Aguirre, E. (1991): «Mandibules et dents d'Ibeas (Espagne) dans le contexte de l'evolution humaine en Europe». L'Anthropologie, 95, 1: 89-102.

Zazo, C., Goy, J. L. y Hoyos, M. (1987): "Contexto Geológico y Geomorfológico de la Sierra de Atapuerca». En E. Aguirre et alii (eds.) (1987): 41-46. 